\title{
Emergent Functional Network Effects in Parkinson Disease
}

\author{
Caterina Gratton ${ }^{1}$, Jonathan M. Koller ${ }^{2}$, William Shannon ${ }^{3}$, Deanna \\ J. Greene ${ }^{2,4}$, Baijayanta Maiti ${ }^{1}$, Abraham Z. Snyder ${ }^{1,4}$, Steven \\ E. Petersen ${ }^{1,4,5,6,7,8}$, Joel S. Perlmutter ${ }^{1,4,6,9,10}$ and Meghan C. Campbell ${ }^{1,4}$
}

${ }^{1}$ Department of Neurology, Washington University in St. Louis, St. Louis, MO 63110, USA, ${ }^{2}$ Department of Psychiatry, Washington University in St. Louis, St. Louis, MO 63110, USA, ${ }^{3}$ BioRankings, St. Louis, MO 63108, USA, ${ }^{4}$ Department of Radiology, Washington University in St. Louis, St. Louis, MO 63110, USA, ${ }^{5}$ Department of Psychology, Washington University in St. Louis, St. Louis, MO 63110, USA, ${ }^{6}$ Department of Neuroscience, Washington University in St. Louis, St. Louis, MO 63110, USA, ${ }^{7}$ Department of Biomedical Engineering, Washington University in St. Louis, St. Louis, MO 63110, USA, ${ }^{8}$ Department of Neurological Surgery, Washington University in St. Louis, St. Louis, MO 63110, USA, ${ }^{9}$ Department of Occupational Therapy, Washington University in St. Louis, St. Louis, MO 63110, USA and ${ }^{10}$ Department of Physical Therapy, Washington University in St. Louis, St. Louis, MO 63110, USA

Address correspondence to Meghan C. Campbell, Departments of Neurology and Radiology, Washington University School of Medicine, Campus Box 8111, 4525 Scott Ave., Rm. 2106, St. Louis, MO 63110, USA. Email: meghanc@wustl.edu

\begin{abstract}
The hallmark pathology underlying Parkinson disease (PD) is progressive synucleinopathy, beginning in caudal brainstem that later spreads rostrally. However, the primarily subcortical pathology fails to account for the wide spectrum of clinical manifestations in PD. To reconcile these observations, resting-state functional connectivity (FC) can be used to examine dysfunction across distributed brain networks. We measured FC in a large, single-site study of nondemented PD ( $N=107$; OFF medications) and healthy controls $(N=46)$ incorporating rigorous quality control measures and comprehensive sampling of cortical, subcortical and cerebellar regions. We employed novel statistical approaches to determine group differences across the entire connectome, at the network-level, and for select brain regions. Group differences respected well-characterized network delineations producing a striking "block-wise" pattern of network-to-network effects. Surprisingly, these results demonstrate that the greatest FC differences involve sensorimotor, thalamic, and cerebellar networks, with notably smaller striatal effects. Split-half replication demonstrates the robustness of these results. Finally, block-wise FC correlations with behavior suggest that FC disruptions may contribute to clinical manifestations in PD. Overall, these results indicate a concerted breakdown of functional network interactions, remote from primary pathophysiology, and suggest that FC deficits in PD are related to emergent network-level phenomena rather than focal pathology.
\end{abstract}

Key words: fMRI, functional connectivity, networks, Parkinson disease 


\section{Introduction}

The pathologic hallmarks of Parkinson disease (PD) are $\alpha$-synuclein aggregated Lewy bodies in substantia nigra, degeneration of nigrostriatal dopaminergic neurons, and striatal dysfunction, resulting in the classic motor deficits of PD. However, subcortical dysfunction alone fails to account for the full spectrum of clinical manifestations in $\mathrm{PD}$, which includes cognitive, psychiatric, and other nonmotor disturbances (Jankovic 2008; Aarsland and Kramberger 2015). Although PD neuropathology becomes progressively more complex as $\alpha$-synuclein aggregation extends beyond the brainstem to subcortical structures and eventually cortical regions later in the disease course (Braak et al. 2004; Halliday et al. 2011; Kotzbauer et al. 2012), the clinical presentation of PD does not necessarily follow the spatiotemporal pattern of neuropathology. The functional mechanisms that relate neuropathology and the varied behavioral disturbances in PD remain unclear (Aarsland and Kramberger 2015). One possible mechanism is that neuropathology in PD causes emergent effects on widespread brain networks that underlie these varied behavioral manifestations.

Thus, the distributed nature of neuropathological and clinical effects in PD emphasizes the need to examine the full functional "connectome" to understand the functional consequences of PD, rather than isolated regions or networks. Functional brain networks can be measured with resting-state functional connectivity (FC), the temporal correlations in fMRI blood oxygen level dependent (BOLD) signal between pairs of brain regions (Biswal et al. 1995). This approach has been fruitful in characterizing networks in healthy young (Power et al. 2011; Yeo et al. 2011) and older (Chan et al. 2014) populations.

While a large number of FC studies have been conducted in $\mathrm{PD}$, these studies report highly variable differences in the disease (Tahmasian et al. 2015), likely reflecting differences in scope and methodology (reviewed in Supplementary Table S1). Many FC investigations in PD focused on circumscribed brain regions and networks, concentrating primarily on the striatum as the principle locus for motor-related pathophysiology (Helmich et al. 2010; Hacker et al. 2012; see review by Tahmasian et al. 2015). Others focused on FC of cortical networks (Gottlich et al. 2013; Olde Dubbelink et al. 2014; Baggio et al. 2015; Campbell et al. 2015; Gorges et al. 2015; Peraza et al. 2017) without measuring differences in subcortical systems. Moreover, head motion-even submillimeter-may confound

Table 1 Clinical characteristics. Values represent group means and standard deviations (SD). Two participants were missing MMSE; 3 participants were missing OFF UPDRS and LEDD. MMSE = Minimental State Exam; UPDRS-III OFF = Unified Parkinson's Disease Rating Scale (III), off medication; LEDD = Levodopa equivalent daily dose

\begin{tabular}{lccl}
\hline Clinical characteristics & $\begin{array}{l}\text { PD } \\
\text { Mean (SD) }\end{array}$ & $\begin{array}{l}\text { HC } \\
\text { Mean (SD) }\end{array}$ & $\begin{array}{l}\text { PD vs. HC } \\
\text { P-value }\end{array}$ \\
\hline N & 107 & 46 & - \\
Sex (\% male) & 56.1 & 30.4 & 0.004 \\
Age & $65.2(7.3)$ & $63.8(11.0)$ & 0.37 \\
Years of education & $16.2(2.6)$ & $14.8(2.7)$ & 0.002 \\
MMSE & $28.6(1.6)$ & $29.0(1.1)$ & 0.13 \\
Disease duration (years) & $6.3(4.2)$ & - & \\
Age onset of PD & $59.0(7.6)$ & - & \\
UPDRS-III OFF total & $23.1(8.5)$ & - & \\
LEDD & $792(545)$ & - & \\
\hline
\end{tabular}

Note: Bold values highlight significant group differences.
FC analyses (Power et al. 2012; Satterthwaite et al. 2012; Van Dijk et al. 2012; Ciric et al. 2017); most studies in PD do not adequately address this issue despite being classified as a movement disorder (Supplementary Table S1). Thus, current studies have not provided a thorough examination of the extent and relative magnitude of network-level FC differences in PD across the entire connectome.

To address this need, we take a broad and multiscale approach to measure the effect of PD on functional networks. We analyzed a large and well-characterized PD dataset (107 PD off medication, 46 age-matched healthy controls [HC]) with meticulous attention to data quality and motion artifacts. We measure disruptions to functional networks at multiple levels: the whole connectome, individual networks, and by region, comprehensively sampling cortical, striatal, thalamic, and cerebellar regions. We use split-half testing to examine the reproducibility of our findings. Finally, we examine the relationship of behavior to these functional network differences. This comprehensive approach allows us to robustly determine the relative magnitude and specificity of functional network effects in PD.

\section{Methods \\ Data Collection Overview}

The data included in this article were collected as part of a larger on-going, longitudinal study that acquires MRI, PET, CSF, behavioral, and clinical measures from PD participants and HC with autopsy confirmation and biochemical measures (Foster et al. 2010; Campbell et al. 2015; Lucero et al. 2015; Buddhala et al. 2015a, 2015b). Here we analyze MRI data from nondemented PD and HC participants from the first time-point that met rigorous quality control standards (baseline visit for $N=129$, with the remaining from follow-up visit years 1-6). All PD participants completed neuroimaging, cognitive, and motor assessments after overnight withdrawal of PD medications (i.e., "OFF" meds). Control participants were screened for neurological and cognitive impairment as well as biomarkers of preclinical Alzheimer's disease (i.e., CSF and PET measures of $\beta$-amyloid) (Sperling et al. 2011). The Washington University in St. Louis Institutional Review Board and Radioactive Drug Research Committee approved all procedures; all participants gave written informed consent.

\section{Participants}

A total of 143 participants with PD and 58 HC completed MRI scans. Of these, $107 \mathrm{PD}$ and $46 \mathrm{HC}$ participants were included in our analyses. For PD, 5 participants were excluded for dementia, 19 due to excessive movement, 11 due to coregistration issues, and 1 due to poor brain coverage during scanning; for HC, 5 participants were excluded due to elevated cortical $\beta$-amyloid (Pittsburgh compound B; PIB, (Mintun et al. 2006)) PET levels, 5 due to excessive movement, and 2 due to registration errors (exclusion criteria are described in detail throughout the Methods). See Supplementary Methods for a description of PD and HC inclusion criteria and Table 1 for PD and HC characteristics.

\section{Cognitive and Motor Assessments}

Participants completed an extensive battery of motor and cognitive assessments, off medication, including tests of memory, attention, language, visual-spatial, and executive function, and motor severity using the Unified Parkinson Disease Rating Scale motor evaluation (UPDRS-III) (Fahn et al. 1987). See Supplementary Methods for additional details. 


\section{Image Acquisition and Preprocessing}

MRI data were collected on a $3 \mathrm{~T}$ Siemens Trio (Erlangen, Germany) using a 12-channel head coil. A T1-weighted MPRAGE structural scan was collected from each participant in each scan session with: repetition time $(\mathrm{TR})=2400 \mathrm{~ms}$, echo time $(\mathrm{TE})=16 \mathrm{~ms}$, inversion time $(\mathrm{TI})=1000 \mathrm{~ms}$, flip angle $=8^{\circ}$, with $0.9 \mathrm{~mm}^{3}$ isotropic voxels. A T2-weighted MRI was collected with TR $=3200 \mathrm{~ms}, \mathrm{TE}=455 \mathrm{~ms}$, and $1 \mathrm{~mm}^{3}$ isotropic voxels.

Participants also completed 1-3 resting-state fMRI runs, off medication, per scan session using a gradient echo pulse sequence with: 200 volumes $(7.26 \mathrm{~min}), \mathrm{TR}=2200 \mathrm{~ms}, \mathrm{TE}=$ $27 \mathrm{~ms}$, flip angle $=90^{\circ}$, with $4 \mathrm{~mm}^{3}$ isotropic voxels and 48 axial slices (no gaps; FOV $=256 \mathrm{~mm}^{2}$ ). Three resting-state fMRI runs were acquired for most participants. In some cases, 1 (or, rarely, 2) runs were omitted because of limited participant tolerance, time constraints, or excessive head motion. Coverage included all of the cerebral cortex, cerebellum, and brainstem (1 participant was excluded for poor cerebellar coverage). Participants were instructed to rest quietly with eyes closed and to stay awake. Wakefulness was confirmed verbally after each scan. Participants were monitored during scans; runs with sustained observable motion (e.g., tremors, dyskinesia, or dystonia) were excluded. MRI data underwent standard preprocessing as previously described (Campbell et al. 2015; Fox et al. 2005; Hacker et al. 2012) (see Supplementary Methods).

\section{FC Processing and Quality Control}

Additional preprocessing prior to FC analyses included: 1) spatial smoothing ( $6 \mathrm{~mm}$ Gaussian blur in each direction), 2) lowpass temporal filtering $(<0.1 \mathrm{~Hz})$, and 3$)$ regression of nuisance signals from white matter, ventricles, the global signal, and 6 motion parameters and their derivatives. Global signal regression (GSR) was included to reduce physiological noise generated by head motion and respiration, as these sources of artifacts can substantially contaminate FC data (Power et al. 2012; Satterthwaite et al. 2012; Van Dijk et al. 2012; Ciric et al. 2017) and the preponderance of studies comparing processing strategies have demonstrated that GSR is the best way to remove artifacts for group studies, especially from movement and respiration (Power et al. 2012, 2014, 2015, 2017; Satterthwaite et al. 2012, 2017; Burgess et al. 2016; Ciric et al. 2017). As PD is a movement disorder, GSR is particularly important to properly interpret group differences in FC between PD and HC. Despite the overwhelming evidence for its positive effects, only 3 of the previous 18 studies reviewed in Supplementary Table S1 include GSR as a processing step (and, notably, only 1 of these also includes motion censoring) (Campbell et al. 2015).

\section{Motion Censoring}

FC preprocessing was run (as described above) in 2 passes. Processed data from the first pass was used to identify frames with high frame-to-frame signal intensity changes $(>0.3 \%)$ (Power et al. 2012). In the second pass, these individual frames contaminated by motion were censored before the start of processing. Entire runs were removed from analysis if they had fewer than 50 useable frames or $>1.0 \mathrm{~mm}$ root mean squared head motion across the entire run. Furthermore, participants were excluded if the participant had $<150$ usable frames ( $5.5 \mathrm{~min}$ ) across all collected fMRI runs or a mean frame-toframe displacement (FD, calculated precensoring) $>0.5 \mathrm{~mm} ; 19$
PD and 5 control participants were excluded based on these criteria.

After these steps, PD and HC groups did not differ in levels of motion (mean precensoring $F D, P=0.60$ ). In both groups, less than $0.5 \%$ of functional connections showed significant correlations with QC measures, benchmarking well against current standards for motion correction (Ciric et al. 2017) (see Supplementary Fig. S1). The number of retained volumes did not differ between groups $(P=0.18$; $\mathrm{PD}$ : mean $=457$, range $=$ 159-583; HC: mean $=454$, range: 199-582).

\section{Regions and Networks}

Regions were defined to provide a comprehensive representation of cortical and subcortical regions, while controlling for potential atrophy effects. Regions included previously defined functional cortical parcels (Gordon et al. 2016), thalamic regions derived from a group parcellation method (as in Greene et al. (2014), subject-specific striatal and medial temporal lobe (MTL) regions from FreeSurfer v5.3 (Fischl et al. 2002, 2004), and subject-specific cerebellar regions from SUIT (Diedrichsen 2006; Diedrichsen et al. 2009); (see below for details on region definition). Regions were masked by each participant's gray matter to account for possible atrophy; regions with fewer than 2 voxels for any participant were removed from analysis (66 regions removed; see Fig. 1). We also excluded regions of low BOLD signal that did not cluster well ("unassigned") (Gordon et al. 2016) leaving 285 ROIs. These criteria were included in an effort to provide the cleanest and least biased functional network data for the comparison of $\mathrm{PD}$ and $\mathrm{HC}$ networks, as some regions cannot be studied accurately with MRI because they fall within susceptibility voids or have limited signal due to atrophy. Two HC and 11 PD participants were excluded owing to poor gray/ white segmentations.

\section{Cortex}

Cortical regions $(N=333)$ were from a previously published group parcellation, based on applying a boundary mapping technique on surface-based FC data in a large group of young, healthy, individuals (Gordon et al. 2016). These surface regions were projected into the volume for analysis. Cortical regions were then restricted to each individual's FreeSurfer (v5.3) (Fischl et al. 2002, 2004) gray matter ribbon to account for possible atrophy.

\section{Striatum and MTL}

ROIs in the striatum (8 regions: bilateral caudate, anterior putamen, posterior putamen, and nucleus accumbens) and MTL (4 regions: bilateral hippocampus and amygdala) were defined based on subject-specific segmentations from FreeSurfer. Each of these regions (except nucleus accumbens) was eroded by setting the FreeSurfer-defined ROI voxel intensity to 1.0, smoothing with a Gaussian kernel with FWHM $4.0 \mathrm{~mm}$, and setting voxels less than 0.75 equal to 0.0 . Sections for the anterior and posterior putamen were defined on a 488 atlas template image by defining the anterior-posterior division as the posterior aspect of the fornix on each axial slice (Hacker et al. 2012). These sections were then applied to each participant's putamen ROIs after their T1 image had been warped to t88 space.

\section{Thalamus}

Given the lack of reliable anatomical MRI markers for reliably identifying the small nuclei of the thalamus, we delineated 
A
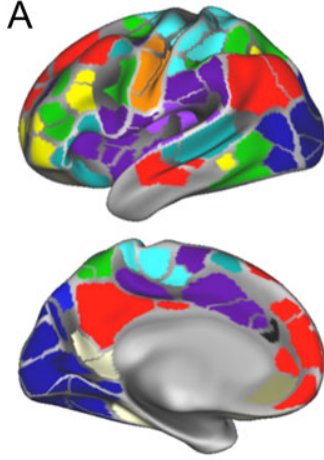

B

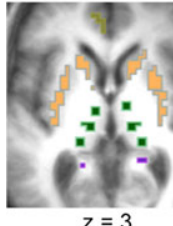

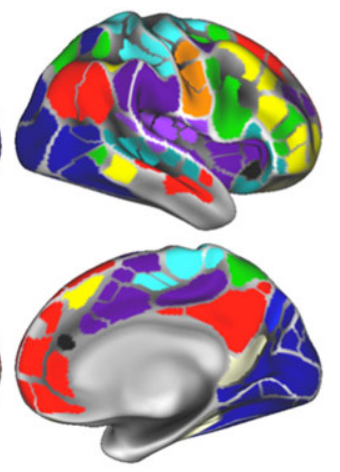
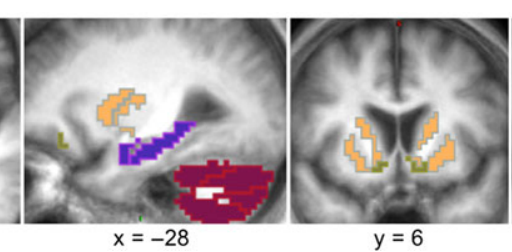
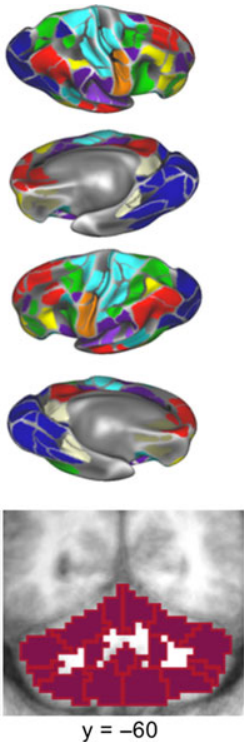

Cortical Sensorimotor Systems

Somatomotor (SM)

Somatomotor lateral (SM-lat)

Visual

Auditory

Cortical Association Systems

Cinguloopercular (CO)

Frontoparietal (FP)

Dorsal Attention (DAN)

Ventral Attention (VAN)

Salience

Default (DMN)

Parietal Memory (Par Memory)

Retrosplenial

Figure 1. Networks and Regions. We examined resting-state correlations in a comprehensive set of cortical and subcortical regions representing 17 distinct networks (A) Cortical networks, defined by cortical parcellation, included sensorimotor and association networks (Gordon et al. 2016). (B) Subcortical networks included striatal, medial temporal (MTL), thalamic, and cerebellar regions.

twelve thalamic regions (8 $\mathrm{mm}$ spherical ROIs) based on a functional parcellation of thalamic areas. The parcellation was generated using a functional network winner-take-all method applied to resting state data from a separate group of adults (see, Greene et al. 2014, for details on the method). Briefly, thalamic parcels were defined according to the cortical functional network that correlated most with each thalamic voxel. We then placed a spherical ROI at the centroid of each proposed parcellation, where we have the best confidence in labeling the function of each region. Based on these parcels, we included 2 regions with representation of the visual network (likely corresponding to bilateral pulvinar nuclei), 4 regions with representation of somatomotor networks (in the area of bilateral ventral nuclei), and 6 with representation of frontal association networks (2 in bilateral dorsal anterior thalamus, 2 in bilateral ventral anterior thalamus, and 2 in bilateral dorsomedial thalamus). Thalamic ROIs were masked by the FreeSurfer generated thalamic region from each participant.

\section{Cerebellum}

Fifteen cerebellar regions were anatomically defined from subjectspecific SUIT segmentations (Diedrichsen 2006; Diedrichsen et al. 2009), optimized for older adult brains by masking CSF from the T2 image and constraining the cerebellar boundary with the FreeSurfer generated cortical ribbon (Myers et al. 2017).

\section{Networks}

Network definitions followed Gordon et al. (2016), representing well-established and validated network architecture (Power et al. 2011), with the addition of 5 subcortical networks: basal ganglia, thalamus, reward (nucleus accumbens and orbitofrontal regions), cerebellum, and MTL. Thus, 17 networks shown in Figure 1 were used for all subsequent analyses.

Network definitions were confirmed in this dataset (Supplementary Fig. S2) using the infomap community detection algorithm (Rosvall and Bergstrom 2008; Power et al. 2011) jointly on all cortical parcels, subcortical, and cerebellar ROIs, on weighted graphs created from $1 \%$ to $10 \%$ edge densities.
This data-driven approach produced similar network definitions for cortical areas as previously defined, including sensorimotor (e.g., somatomotor, somatomotor lateral, visual, and auditory) and association (e.g., default mode, salience, cinguloopercular, frontoparietal, dorsal attention, and ventral attention) networks. In addition, subcortical regions clustered into 5 networks: basal ganglia, thalamus, reward (nucleus accumbens and orbitofrontal regions), cerebellum, and medial temporal lobe.

\section{Spherical Parcellation}

As an additional test of whether effects were consistent across cortical parcellations and with regions of matched size, 300 8-10 mm spherical ROIs (Power et al. 2011; Greene et al. 2014; Seitzman et al. 2017) with coverage across cortex, striatum, thalamus, MTL, and the cerebellum were used to examine the pattern of functional network differences in PD. Cortical ROIs were spheres from Power et al. (2011) and subcortical ROIs were defined as for the thalamus above (Greene et al. 2014). No gray matter masking was performed on these spherical ROIs.

\section{FC Calculation}

FC was calculated as Fisher z-transformed temporal correlations between pairs of averaged BOLD signals from each region. Results were assembled into region $\times$ region matrices, sorted by network assignment. Sets of correlations between a given pair of networks (or within the same network) are referred to as "blocks" in this matrix structure. These FC matrices define an unthresholded weighted graph that represents the organization of functional networks across the whole brain, in which regions are represented as nodes, and FC values are represented as weighted, undirected, edges.

\section{Connectome and Network-Level Analyses}

Principal statistical results were obtained by comparing PD and HC groups using a novel technique for contrasting 
connectomes (object-oriented data analysis [OODA] (La Rosa et al. 2012, 2016); see below). To test the robustness of our methods, we repeated OODA on random split-half samples of our data. We then conducted post hoc comparisons using a permutation approach (see below) to assess which network blocks showed significant group differences. We also examined more traditional correlation maps obtained using seeds in key regions (e.g., striatum). Finally, we examined how post hoc selected network blocks relate to cognitive and motor performance, partialing out the effects of age, sex, and education. A more complete description of these analyses is below.

\section{Object Oriented Data Analysis}

A weighted graph OODA method (La Rosa et al. 2012, 2016) was used to test whether correlation matrices differ systematically between PD and HC groups. OODA is a multidimensional comparison of the differences between whole matrix objects that preserves individual edges and their ordering within the matrix in comparisons (conceptually related to comparing the similarity of the upper triangle of matrices). Specifically, in this approach, individual correlation matrices are treated as a single object. Average weighted $\left(G^{*}\right)$ matrices from each sample are computed under the assumption that the matrices follow the Gibbs distribution. Average matrices are then compared with one another by taking the Euclidian distance between them (i.e., by computing the Euclidean distance between the upper triangles of each average matrix). To assign a $P$-value to the observed differences, a distribution of distances is generated by bootstrapping the samples ( $N=1000$ times) in each original dataset to create new average $G^{*}$ matrices. The OODA method reduces the need for substantial data reduction in the correlation matrices by allowing full matrices to be compared, rather than individual networks or connections, thereby obviating the loss of power from testing each connection separately. Thus, OODA provides an omnibus-level statistical test of the entire graph (i.e., correlation matrix).

\section{Multidimensional Scaling}

Multidimensional scaling (MDS) plots were used to represent the similarity among matrices based on their distances (computed as the Euclidian distance between matrix objects). In MDS, data points are represented in an $\mathrm{N}$-dimensional space, computed to best preserve the relative distances between each point (note that absolute values/directions are not meaningful in this representation); the initial dimensions in MDS represent the primary variance in the data. We use the classical MDS algorithm implemented in the Matlab 2012 cmdscale.m function, applied to the full set of PD and HC participants.

\section{Matched Group Analyses}

An additional control analysis was conducted on a sub-sample of PD participants matched to HC on sample size, sex, age, and years of education. The matched samples were statistically compared with one another using the OODA method described above.

\section{Split-Half Analyses}

To determine the robustness of our findings, we conducted additional analyses on a split-half sample of our dataset. PD and HC participants were randomly split into 2 halves (for each half, $N_{\mathrm{PD}}=54, \mathrm{~N}_{\mathrm{HC}}=23$ ) and statistically compared with one another using the OODA statistic described above. The process was repeated for 50 random splits of the data.

\section{Post Hoc Permutation Testing}

We used a permutation approach to determine which networkto-network blocks differed significantly between PD and HC groups. Group identity labels from the participants were randomly permuted ( $N=1000$ times) and these new permuted assignments were used to generate 1000 novel average "PD" and "HC" matrices. We then measured the average absolute FC difference for each block between the permuted PD and HC matrices. The true PD versus HC difference was contrasted with this block-specific permuted distribution of differences to generate a $P$-statistic. We corrected $P$-values for multiple comparisons across blocks using the FDR approach (requiring that $P(F D R)<0.05$ ).

\section{FC Seed-Based Correlation Maps}

Seed-based correlation analyses were used to study the topography of FC differences in greater detail and to contrast with previously published results. Specially, we calculated seed-based FC correlation maps, focused on some of the primary regions of differences, including cortical motor areas, thalamus, and striatum given previous papers focused on this area. As seeds, we used the same ROIs as defined above. To create a seed-based correlation map, the average signal for a given ROI was correlated with signals from every other voxel in the brain. These maps were Fisher z-transformed and averaged across participants in a group. Cortical motor and thalamic correlation maps were created for display purposes, to explore the pattern of FC differences across cortical and subcortical structures. For striatal correlation maps, we additionally conducted Monte Carlo permutation-based cluster correction $(N=10000$; for $t>3.0$, FWE $P<0.05)$ to directly compare our findings with previous literature on striatal FC differences in PD.

\section{Relationship to Behavior}

Finally, we examined whether mean FC in the selected blocks relate to behavioral performance. For each block, we examined the correlation between the average FC in that block with cognitive (attention, memory, language, visuospatial, executive, and average cognitive performance) and motor (UPDRS-III total score, and bradykinesia, tremor, rigidity, and PIGD UPDRS-III subscores) scores, partialling out age, sex, and education. Partial-correlation P-values were FDR corrected for multiple comparisons across selected blocks.

\section{Results \\ Overview}

We examined large-scale, whole-brain networks in 107 nondemented PD (off-medication) and 46 HC participants. First, we demonstrate functional connectome-level differences between PD and HC participants using novel omnibus-level statistical methods (OODA). We show that these differences are robust, replicating in sub-samples of our data, and not driven by sample characteristics. FC differences-primarily reductions in (positive and negative) FC magnitude-were widespread, but highly selective to particular network-to-network blocks, within and between cortical and subcortical networks. We present a detailed analysis and ranking of these "block-wise" network effects, and, finally, show that a subset of FC blocks relate to motor and cognitive performance. 


\section{Participant Characteristics}

PD and HC participant characteristics are shown in Table 1. PD and HC groups were matched on age, however, PD participants had more education $(P<0.005)$, and a higher proportion of males than HC participants $\left(\chi^{2}=8.47, \mathrm{df}=1, \mathrm{P}<0.005\right)$. See analyses below (Evaluation of FC Differences: Matched Groups) to address this issue; additionally, sex, age, and education were included as covariates for FC-behavior analyses.

Although not demented, PD participants performed worse than HC participants across many cognitive domains, including attention, memory, and executive function (Supplementary Table S2). Thus, this was a heterogeneous sample, with a range of motor and cognitive features typical of PD.

\section{Connectome Characteristics}

We measured large-scale functional networks in PD and HC across cortical, subcortical, and cerebellar regions comprehensively representing the functional connectome (Fig. 1). Both groups showed similar network organization (Fig. 2A), that is, both matrices exhibited high within- and low between-network correlations. Indeed, similar network divisions were found in both groups with data-driven community detection algorithms (Supplementary Fig. S2) largely consistent with networks identified in young HC (Gordon et al. 2016). However, direct group comparison reveals widespread and selective block-level FC differences between PD and HC participants (Fig. 2B), impacting a combination of cortical sensorimotor, subcortical, and association networks. In these blocks, PD participants demonstrated decreased positive connections and increased negative connections in $\mathrm{PD}$, corresponding to a reduced absolute magnitude of FC.

\section{Connectome-Level Differences}

We applied graph-based statistical techniques to determine whether these observed FC differences significantly differed between PD and HC. The groups clustered separately from one another along the first 3 MDS dimensions, qualitatively
A

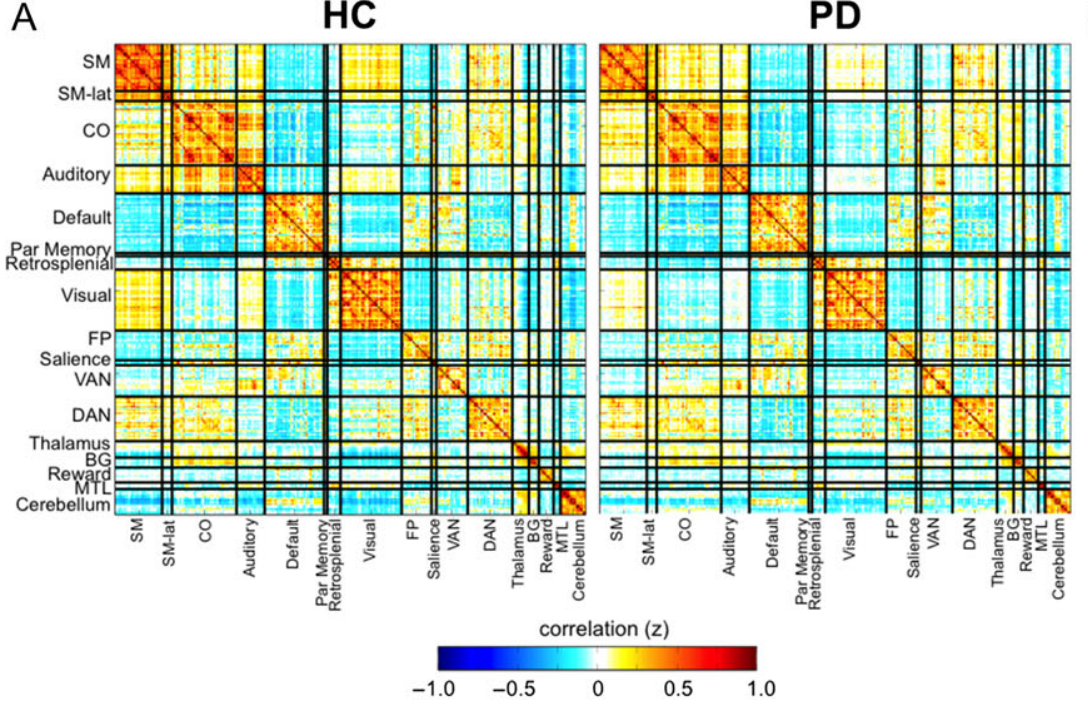

B

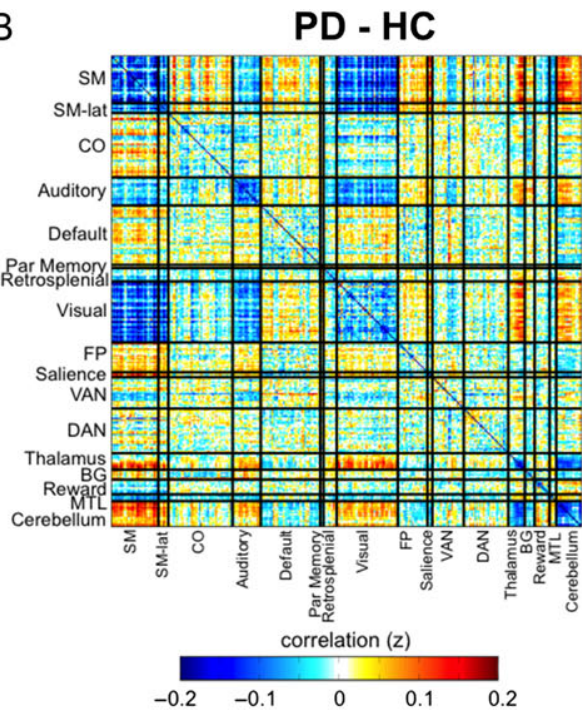

C
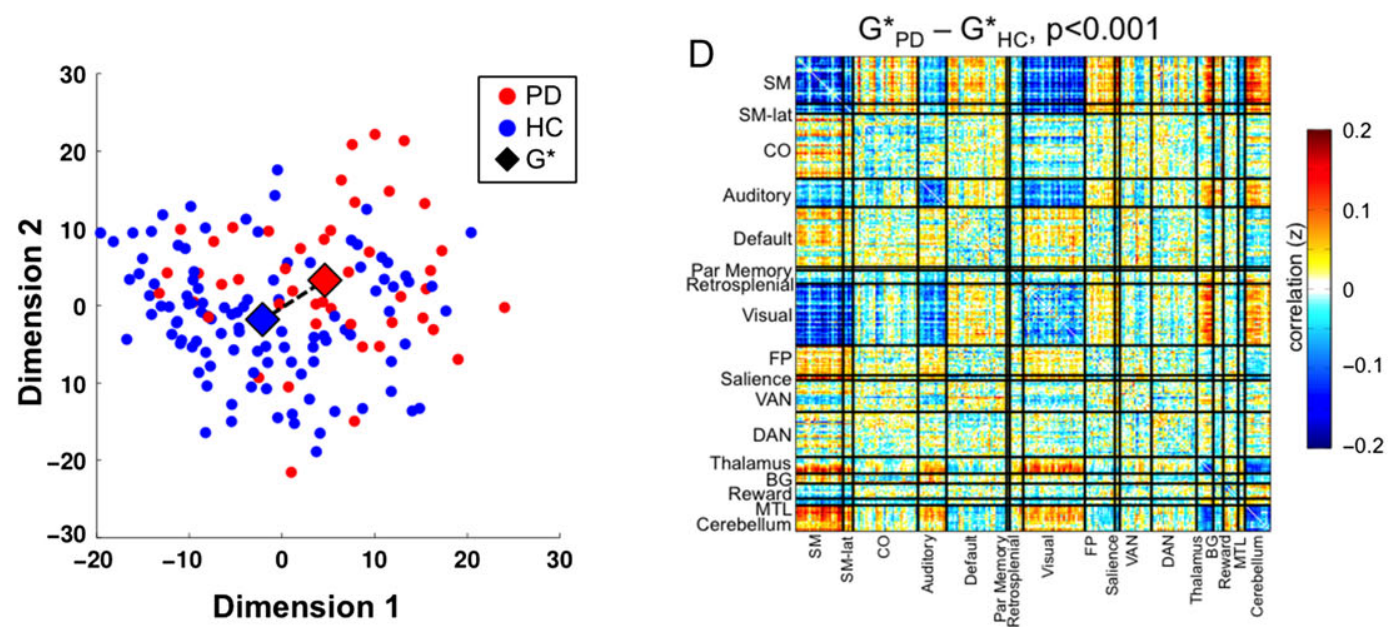

Figure 2. Large-scale networks in Parkinson disease and controls. (A) Large-scale networks in HC (left) and PD (right) participants, shown in the form of a correlation matrix. Both PD and HC participants showed strong network organization, with high correlations within each network (diagonal) and low correlations between networks (off-diagonals). (B) Direct comparison between groups shows prominent and highly structured differences characterized by a block-specific pattern. (C) Multidimensional scaling plot shows the relative positions of PD (blue) and HC (red) matrices in multidimensional space. Large diamonds represent the central tendency of each group $\left(G^{*}\right)$, determined using the Gibbs distribution. The dashed line marks the distance between the $2 G^{*}$ matrices. (D) The difference between $G_{P D}{ }^{*}$ and $G_{H C}{ }^{*}$ matrices using OODA is depicted, with significance assessed by bootstrapping $(P<0.001)$. 
demonstrating distinct FC distributions between groups (Fig. 2C; each point represents a full FC matrix). We used OODA to quantify group differences. OODA identifies the average graph of a sample $\left(G^{*}\right)$. Differences between 2 average graphs then can be quantified by calculating the distance between them in multidimensional space. Finally, bootstrapping was used to determine the significance of this distance. As shown (Fig. 2D) PD and HC connectomes differed significantly $(\mathrm{P}<$ 0.001), with the difference in $G^{*}$ matrices reproducing a similar pattern to the simple differences shown in Figure $2 B$. Similar results were also seen with a different parcellation based on 300 spherical regions of matched size (OODA $P<0.001$; Supplementary Fig. S3).

\section{Matched Groups}

Given that the PD and HC groups differed in number, sex ratio, and education, we conducted a supplementary analysis with a subsampling of PD participants to match the groups on these variables (Supplementary Fig. S4). OODA indicated that the matched groups were significantly different $(P<0.001)$; furthermore, the distribution of differences was analogous to those shown in Figure 2.

\section{Reliability of FC Results}

Finally, given the novel techniques employed in the current study, we used a split-half procedure to evaluate the reliability of our findings. PD and HC participants were randomly split into 2 equal halves $\left(\mathrm{N}_{\mathrm{HC}}=23, \mathrm{~N}_{\mathrm{PD}}=54\right.$ per half) and compared qualitatively as well as quantitatively using OODA (Supplementary Fig. S5). Despite the smaller sample sizes, results were similar for the 2 halves, with significant PD versus HC OODA differences per each half (Supplementary Fig S5A shows the distribution of $P$-values across 50 random splits, mean $\pm S D, P=0.02 \pm 0.03$, median $P=0.008$ ). The difference in $G^{*}$ matrices (Supplementary Fig. S5B, shown for the median split-half result) demonstrates a similar pattern to the one described above, with prominent block-specific FC differences between PD and HC participants.
Thus, these results indicate robust network-wide FC differences between PD and HC.

\section{Block-Level FC Differences}

We next used permutation testing to identify network blocks with significant average absolute FC differences between PD and HC participants $(P<0.05$, FDR corrected for multiple comparisons across blocks). Significant blocks are shown in Figure $3 \mathrm{~A}$ and listed in order of magnitude difference in Table 2.

The networks affected in PD generally showed lower magnitudes (whether positive or negative FC) compared with HC; in other words, a shift towards zero. Thus, the dominant pattern of differences was a reduced magnitude of FC within and between affected networks (Fig. 3B, Supplementary Fig. S6).

Although widespread, FC differences were selective and systematically organized, aligning with specific network blocks involving cortical and subcortical regions (Fig. 3). The most pronounced differences occurred within and between cortical sensorimotor, thalamic, and cerebellar networks. A more modest and complex pattern of differences existed between these networks and association networks. Notably, association network impact in nondemented PD was limited to these interactions; there were no differences within or among association networks. Interestingly, blocks involving the striatum ("BG" in Figs 2 and 3) exhibited less prominent FC differences, suggesting that functional differences were downstream of the structures generally thought to be the main sites of dysfunction in PD.

We describe the patterns of FC differences in detail here. Prominent relationships (e.g., among cortical somatomotor, thalamus, cerebellum) were explored further with seed-based FC to determine the spatial distribution of effects and how they align with motor- and nonmotor subregions of subcortical systems. Descriptions of group differences refer to differences in absolute magnitude of FC in PD compared with HC (i.e., magnitude enhancement or reduction, whether from positive or negative, in PD relative to $\mathrm{HC}$-as opposed to a directional increase or decrease in FC), except where specifically noted otherwise.

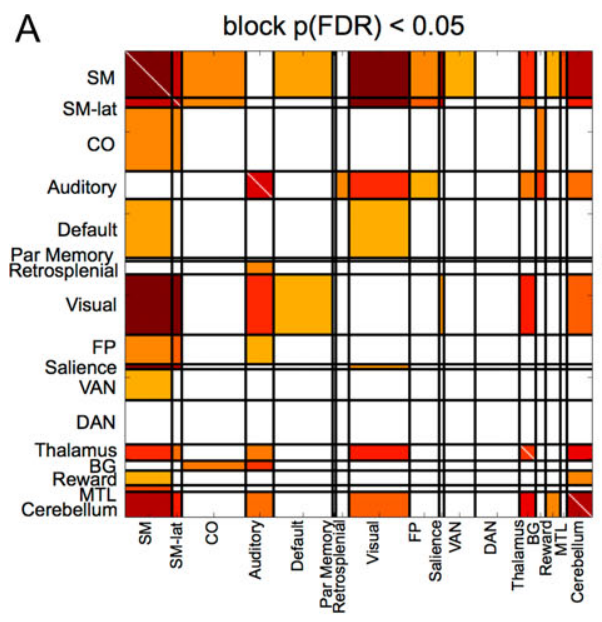

mean abs. correlation difference $(z)$

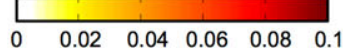

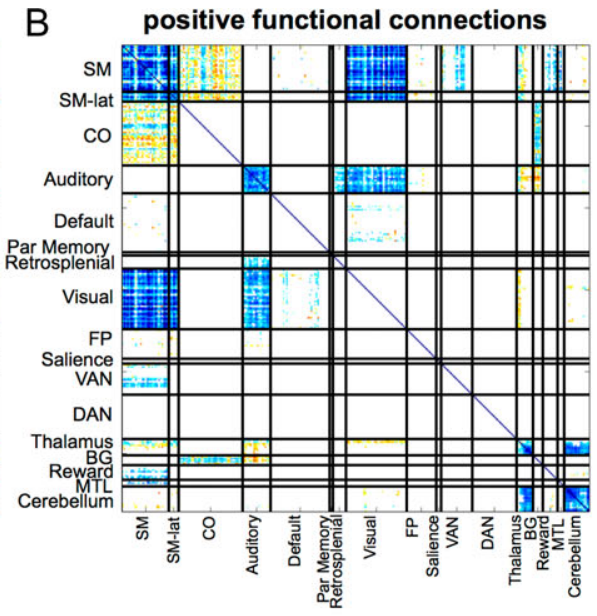
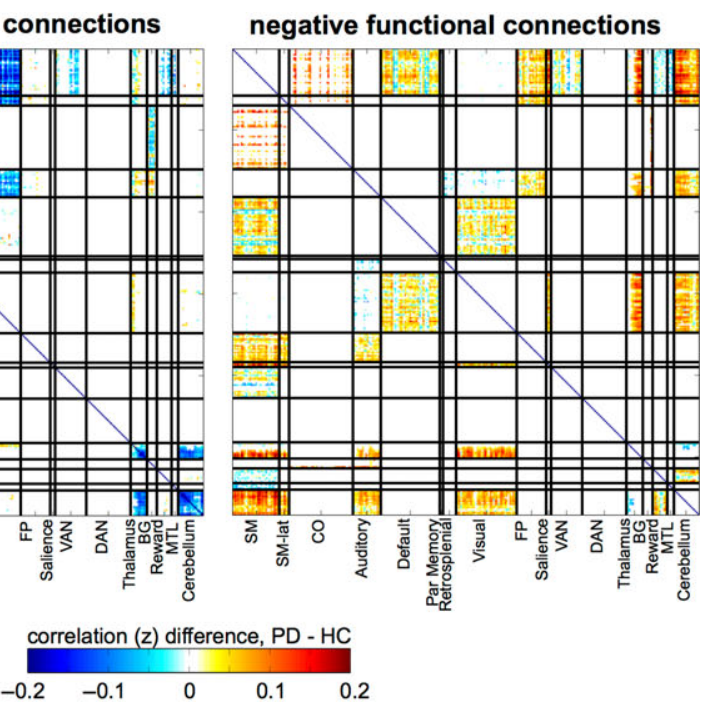

Figure 3. Significant Block-Level Differences between PD and HC. (A) We permuted participant group labels to identify blocks with significant mean absolute differences between PD and HC groups $(\mathrm{P}<0.05$, FDR correcting for the number of blocks). Significant blocks are shown in hot colors, as the absolute difference in FC per block. See Table 2 for a ranking of blocks. (B) Here, we separately show the PD-HC differences of significant blocks, for connections that were positive in HC (left) and negative in HC (right). Most positive connections decrease and most negative connections increase in PD relative to HCs, indicating a reduction in FC magnitude (Supplementary Fig. S6). 
Table 2 Block difference ranking. This table reports the blocks with the largest absolute FC differences between PD and HC groups for all significant blocks (FDR-corrected $P<0.05$ ). The second column reports the number of standard deviations away from the null (permuted) mean and the third column reports the mean absolute FC difference within the block.

\begin{tabular}{|c|c|c|c|}
\hline FDR-Pval & STDperm & Mean & Names \\
\hline 0 & 11.34 & 0.1058 & SM-Visual \\
\hline 0 & 11.24 & 0.1025 & SM-SM \\
\hline 0 & 10.8 & 0.0886 & SM-Cerebellum \\
\hline 0 & 8.53 & 0.1064 & SM-lat-Visual \\
\hline 0 & 8.2 & 0.0944 & SM-Salience \\
\hline 0 & 8.02 & 0.0901 & Cerebellum-Cerebellum \\
\hline 0 & 7.54 & 0.0686 & Auditory-Visual \\
\hline 0 & 6.83 & 0.0695 & Visual-Thalamus \\
\hline 0 & 6.32 & 0.0479 & $\mathrm{CO}-\mathrm{BG}$ \\
\hline 0 & 6.05 & 0.0682 & SM-Thalamus \\
\hline 0.001 & 6.31 & 0.0545 & SM-lat-FP \\
\hline 0.001 & 6.05 & 0.0807 & Thalamus-Cerebellum \\
\hline 0.002 & 6.27 & 0.0864 & SM-SM-lat \\
\hline 0.002 & 6.14 & 0.0462 & SM-FP \\
\hline 0.002 & 5.8 & 0.0688 & SM-lat-Cerebellum \\
\hline 0.002 & 5.79 & 0.0868 & SM-lat-Salience \\
\hline 0.003 & 6.14 & 0.0836 & Auditory-Auditory \\
\hline 0.006 & 5.55 & 0.0626 & Auditory-BG \\
\hline 0.007 & 4.96 & 0.0364 & Auditory-FP \\
\hline 0.011 & 4.42 & 0.0366 & SM-VAN \\
\hline 0.016 & 4.69 & 0.0451 & Reward-Cerebellum \\
\hline 0.017 & 4.86 & 0.0508 & Auditory-Cerebellum \\
\hline 0.017 & 4.74 & 0.0547 & Visual-Cerebellum \\
\hline 0.017 & 4.54 & 0.0465 & $\mathrm{SM}-\mathrm{CO}$ \\
\hline 0.02 & 3.81 & 0.0627 & Thalamus-Thalamus \\
\hline 0.022 & 4.38 & 0.0624 & SM-MTL \\
\hline 0.022 & 3.79 & 0.089 & SM-lat-SM-lat \\
\hline 0.028 & 3.99 & 0.0491 & Auditory-Thalamus \\
\hline 0.028 & 3.63 & 0.0528 & SM-lat-Thalamus \\
\hline 0.035 & 3.89 & 0.0402 & SM-Default \\
\hline 0.035 & 3.67 & 0.0364 & SM-Reward \\
\hline 0.035 & 3.52 & 0.0344 & Default-Visual \\
\hline 0.044 & 3.45 & 0.0439 & Auditory-Retrosplenial \\
\hline 0.05 & 3.34 & 0.0426 & Visual-Salience \\
\hline
\end{tabular}

- Within-network FC: The magnitude of intranetwork FC was reduced in cortical somatomotor (SM), somatomotor-lateral (SM-lat), auditory, thalamic, and cerebellar networks. These FC reductions indicate decreased integrity of these cortical sensorimotor and subcortical networks. Notably, striatal FC differences were not significant (see Striatal FC section below).

- Between-network cortical-to-cortical FC:

- Cortical sensorimotor FC: FC magnitude was strongly reduced between somatomotor networks and sensory cortical networks (especially visual, and to a lesser extent, auditory; Figs 2 and 3; shown for a motor seed in Fig. 4A). FC also was diminished between visual and auditory networks. These differences represent a reduced FC magnitude among typically coherent sensorimotor regions.

o Cortical sensorimotor-association FC: Somatomotor (and, to a lesser extent, visual and auditory) networks exhibited moderate to large magnitude reductions of negative correlations with salience and FP networks (Figs 2 and 3, 4A). More mixed effects were seen among SM and the CO, DMN, and VAN networks (Fig. 3B).

- Between-network cortical-to-subcortical FC: Significant differences were also observed in the interactions between sensorimotor
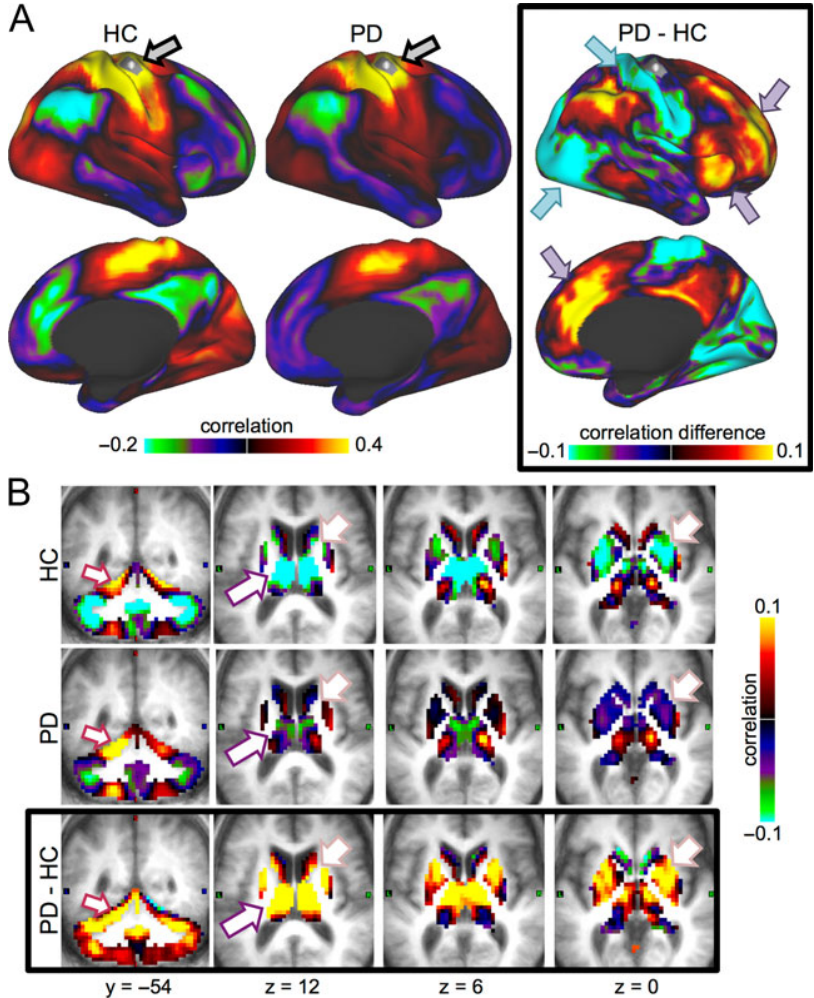

Figure 4. Motor hand seed. We present correlation maps for a motor seed (region shown with a gray patch, indicated with gray arrow in A). (A) Motor cortex FC is projected onto an inflated cortical surface for HC (left), PD (middle), or the group difference (right). PD was associated with decreases in motor FC to sensorimotor and visual regions (cyan arrows) and increases to Salience/CO and FP regions (purple arrows). These differences represent weaker FC in PD relative to HC. Only the right hemisphere is shown, but FC differences were bilaterally symmetric. (B) The same comparisons are shown for volumetric views of subcortical regions. In PD relative to $\mathrm{HC}$, motor cortex FC increased to the cerebellum, especially in motor subregions (indicating stronger FC, maroon arrows). In contrast, motor cortex FC showed little difference to motor subregions of the thalamus, but increased substantially in association-related thalamic subregions (purple arrows; Fig. 5). Motor cortex FC also weakly increased to the striatum, especially in nonmotor subregions of the caudate and anterior putamen (pink arrows; see Supplementary Fig. S7). As with cortical FC, thalamic and striatal FC differences were associated with weakened FC magnitude in PD. Motor cortex FC differences were consistent across sensorimotor regions.

network FC and several subcortical networks, most prominently cerebellar and thalamic (Fig. 3). Interestingly, however, the regional pattern of differences within each structure varied as shown in detailed seed-based FC analyses (Fig. 4B); similar patterns were observed in sensory networks.

o Cortical-Thalamic FC: Dorsal anterior thalamic subregions typically associated with frontal association areas (JohansenBerg et al. 2005; Zhang et al. 2010; Hwang et al. 2017) exhibited prominent reductions of negative FC magnitude in PD (see seed-based FC in Figs 4 and 5; note the thalamic location with high FC to motor cortex in HC shows little difference). Similar differences occurred in thalamic FC with cortical sensory networks.

o Cortical-cerebellar FC: Sensorimotor cortical-to-cerebellar FC differed throughout the cerebellar lobules in PD. In contrast to the thalamus, seed-based analyses reveal that motor subregions of the cerebellum exhibit enhanced positive FC in PD (Fig. 4B). Other subregions exhibited smaller magnitude differences, in this case reduced magnitude of negative FC. 
A

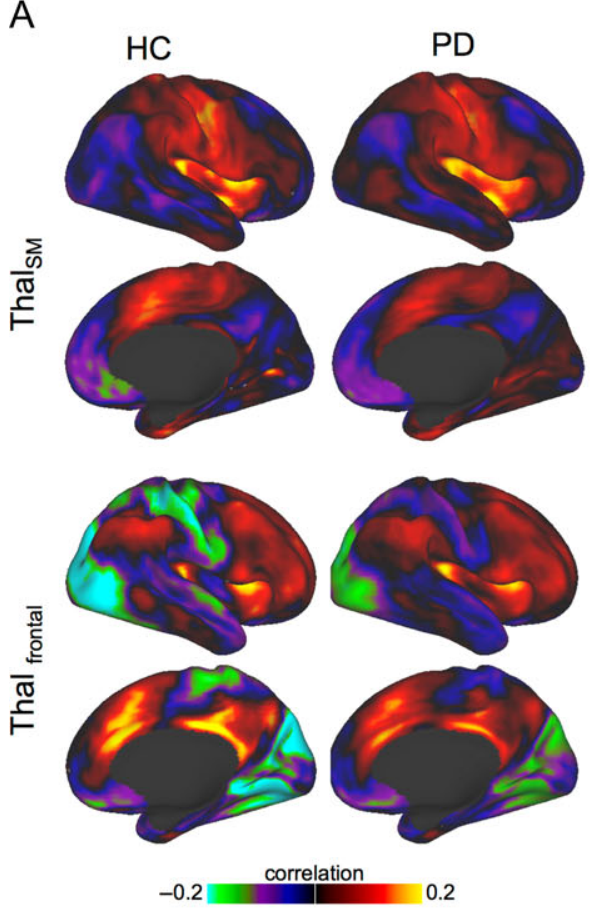

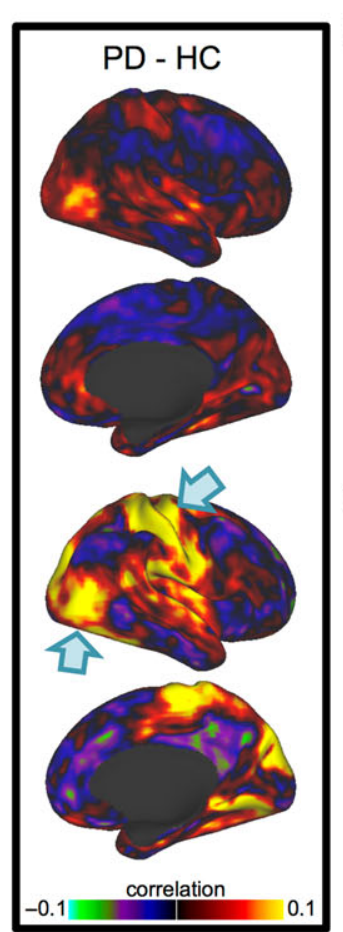
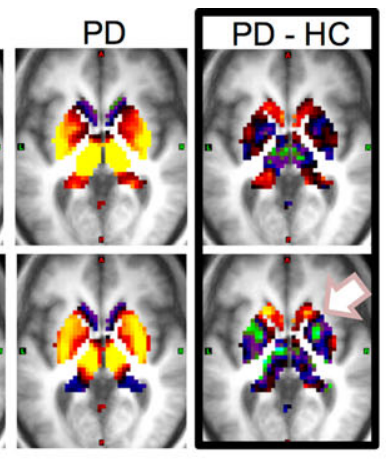

seeds
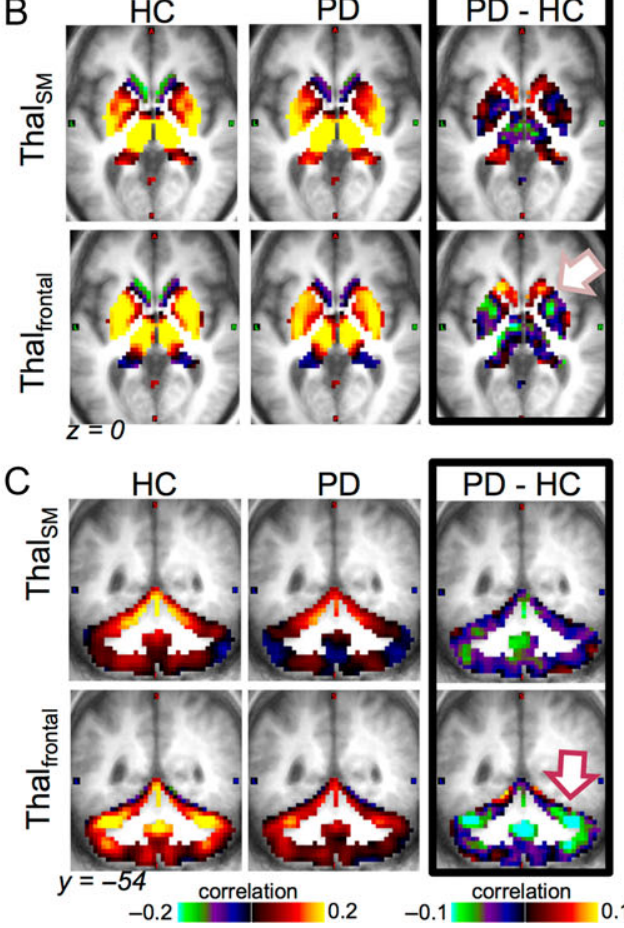

Figure 5. Thalamic seeds. Thalamic FC is shown for 2 thalamic seeds: a seed centered on somatomotor subregions (top row) and a seed centered on frontal association subregions (bottom row; see seeds in gray to the right of B). Columns represent the HC, PD, and group difference FC maps, respectively. Cortical (A), striatum/thalamus (B), and cerebellum (C) differences are shown. The strongest thalamic differences were for frontal thalamic subregions, rather than motor thalamic subregions. This included increases between thalamic subregions and sensorimotor and visual cortex (cyan arrows), decreases to the cerebellum (maroon arrow), and mixed effects in the striatum, with increases to the caudate and decreases to anterior putamen (pink arrow).

o Cortical-other subcortical FC: In PD, stronger (i.e., increased magnitude) negative FC was also present between sensorimotor networks and the MTL and reward networks, indicating enhanced anticorrelations between these regions.

- Subcortical-to-subcortical FC: Reduced FC occurred between cerebellar and thalamic networks, indicating a loss of coherence. In addition, PD showed a reduced magnitude of negative correlations between cerebellum and reward networks.

- Striatal FC: Differences in the striatum were generally smaller than those in the cerebellum, thalamus, and cortical sensorimotor networks, only reaching significance in the BGAuditory and BG-CO blocks. To compare our findings with previous striatal FC results, we conducted focused seed-based striatal FC analyses (Supplementary Fig. S7). These results confirm previously published findings of altered striatal FC with cortical somatomotor and association (especially $\mathrm{CO}$ ) networks, and, to a lesser extent, cerebellar and visual regions (Hacker et al. 2012; Campbell et al. 2015). As shown in Figure 4 and Supplementary Figure S7, sensorimotor-striatal FC demonstrated a similar pattern as the thalamus, with the strongest FC magnitude reductions for nonmotor striatal regions (anterior putamen, caudate). Thus, our results suggest that striatal FC effects in PD are smaller in magnitude compared with FC differences in other subcortical and cortical networks.

These selective intranetwork and internetwork alterations are illustrated in Figure 6, in which connectomes are represented as graphs. Node positions were determined using a springembedding algorithm, which positions more strongly functionally connected nodes closer together. Note the altered size and positions of somatomotor (teal), visual (navy), thalamic (burnt orange), and cerebellar (dark red) networks, which, in PD are larger, pushed farther apart from one another, and interdigitated with other (DAN, CO) networks. This depiction recapitulates the selectively disrupted network integrity-and cross-network interactions-in PD.

\section{Relationship Between FC and Behavior}

Finally, to determine whether FC differences relate to clinical manifestations in $\mathrm{PD}$, we examined the relationships between motor and cognitive performance with FC for each significant block (partialing out the effects of age, sex, and education), correcting for multiple comparisons. UPDRS-III measures of motor severity in PD participants (off medication) correlated with the magnitude of FC, with greater motor severity related to decreased magnitude of negative SM-Reward FC (UPDRS-III total score: partial- $r=0.32, P(F D R)<0.05$; bradykinesia subscore: partial- $r=0.35, P(F D R)<0.05)$ and decreased magnitude of positive FC within the thalamus (UPDRS-III total score: partial- $r=$ $-0.28, P(F D R)=0.08$; bradykinesia subscore: partial- $r=-0.32$, $P(F D R)<0.05)$. Other blocks showed similar relationships but did not reach significance after multiple comparisons correction. These findings suggest that motor dysfunction relates to reduced FC of motor-related cortical and subcortical networks (Supplementary Fig. S8).

Cognitive measures showed more complex relationships within and across networks, not limited to motor-related networks, and differed across cognitive functions (Supplementary Fig. S9). Global cognition scores did not relate to block-level FC, but a subset of cognitive domains correlated with mean block 


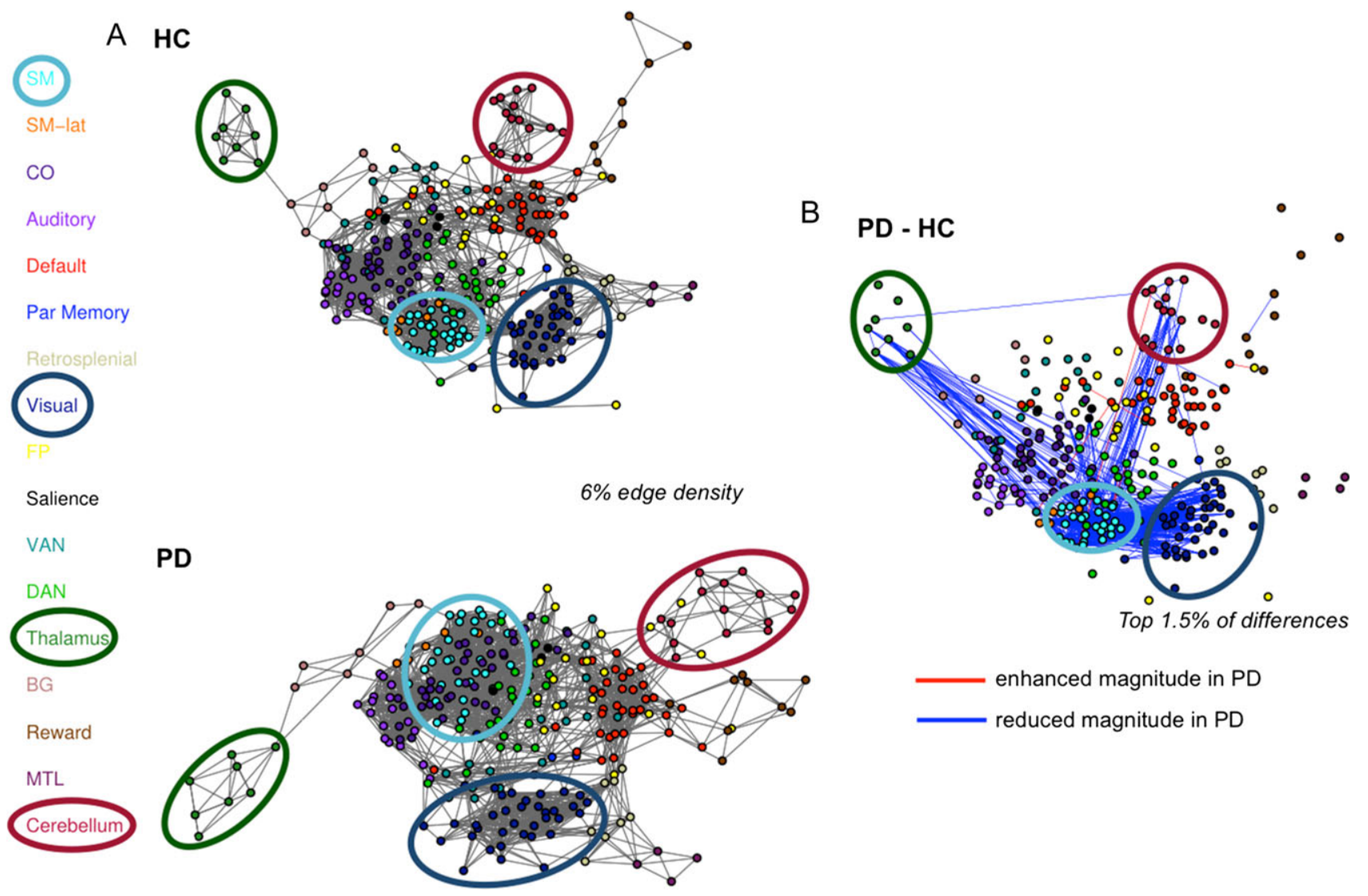

Figure 6. Spring Embedded Graphs. (A) Graph depictions of networks in HC (top) and PD (bottom; shown for 6\% edge density). Brain regions are shown as nodes (filled circles, colors indicate network identity), and edges between nodes indicate functional connections. (B) We highlight the strongest FC differences between PD and HC (red lines = increased FC magnitude for PD, blue lines = decreased FC magnitude for PD; shown for the top 1.5\% of differences; nodes are positioned as in the top graph in A). Strong FC reductions appeared within and between sensorimotor, cerebellar, and thalamic networks. A few selective increases were present between SM and association networks (CO, FP, Salience) and the cerebellum.

FC in the full group of PD and HC participants. Specifically, impaired visuospatial performance was associated with reduced magnitudes of positive intracerebellar FC (partial- $r=$ $0.25, P(F D R)<0.05)$ and negative cerebellar-visual FC (partial- $r=$ $-0.26, P(F D R)<0.05)$. Cerebellar-reward FC flipped from primarily negative in $\mathrm{HC}$ to positive in $\mathrm{PD}$, in association with lower visuospatial performance (partial- $r=-0.24, P(F D R)<0.05$ ). These findings suggest that interactions within and between the cerebellar network and other networks may be central to visuospatial function. Executive function scores also positively related to FC between the SM and MTL networks (partial- $r=$ $0.27, P(F D R)<0.05)$, with better performance associated with a switch from negative to increasingly positive FC.

\section{Discussion}

PD produces a striking "block-wise" pattern of network-level disruptions in FC. Surprisingly, the striatum was not the most significantly affected network; instead, the most prominent disruptions involved interactions among cortical sensorimotor, thalamic, and cerebellar networks. Split-half replication demonstrates the robustness of these results. Thus, in PD, FC disruptions impact specific network-to-network blocks, rather than a regionally limited pattern of differences or global desegregation. Our comprehensive whole-brain sampling combined with novel statistical approaches demonstrates new insights into the extent and relative impact of PD on network-level FC. Overall, these results indicate a concerted breakdown of functional network interactions, remote from primary pathophysiology, suggesting emergent network-level phenomena rather than deficits attributable to focal pathology.

\section{PD is Associated With Selective Network-Level Vulnerabilities}

We find that the pattern of differences between PD and HC is strikingly block-wise, influencing specific sets of network-tonetwork connections, within and between systems. Whereas other studies focused on limited sets of networks, within network alterations, or global measures of FC (see review (Tahmasian et al. 2015); Supplementary Table S1), our results highlight the importance of widespread, but selective, deficits in network interactions. These findings suggest that dysfunction in PD may occur fundamentally at a network level, impacting swaths of vulnerable network-to-network relationships.

Disrupted FC in PD appears distinct from the pattern of FC changes in healthy aging, which are more widespread, less block-specific, and centered on association networks (Chan et al. 2014). Thus, FC reductions with PD do not simply reflect an exaggerated aging process. Whether this pattern of block-wise FC differences is specific to PD or represents a general pattern common to neurodegenerative diseases, including Alzheimer 
disease $(A D)$, remains to be determined. Some findings suggest that dysfunction in neurodegenerative diseases map onto distinct large-scale networks (e.g., DMN in $\mathrm{AD}$, salience in frontotemporal dementia) (Buckner et al. 2005; Seeley et al. 2009). Alternatively, given some shared pathology (e.g., $\beta$-amyloid deposition) and clinical features (e.g., cognitive decline), a similar pattern may exist among diseases, but with differences in the relative prominence and magnitude of the network effects. The end-stage presentation may converge and produce global, cascading failure of networks.

Generally, the distributed nature of FC differences emphasizes the need for whole-brain examination, rather than a focus on a circumscribed set of regions or networks, to understand functional deficits in PD and other neurodegenerative diseases. We applied newly developed graph-based statistical methods (i.e., OODA) that improve the sensitivity of network analyses by computing statistical inferences on the full connectome. This powerful approach provides a more detailed, quantitative characterization of regional, network, and connectome-level FC, which may prove widely useful in network neuroscience.

\section{PD Impacts Specific Cortical and Subcortical Networks and, Especially, Their Interactions}

The most prominent FC differences in PD involved cortical sensorimotor, thalamic and cerebellar networks, both within and, especially, between networks as highlighted by the relative ranking of FC blocks (Table 2). Thus, unlike previous studies that focused largely on the striatum or a few cortical networks (Tahmasian et al. 2015), we show a breadth—but selectivity—of network differences that jointly impact cortical and subcortical systems. The prominent loss of interactions between these networks in PD suggests that PD may lead to a loss of appropriate internetwork integration, as implicated by previous studies investigating internetwork FC (Baggio et al. 2015; Campbell et al. 2015; Peraza et al. 2017).

For example, dysfunction of somatomotor networks is not surprising, given the characteristic motor manifestations of PD (Jankovic 2008) and previous reports of their disruptions (Hacker et al. 2012; Gottlich et al. 2013; Olde Dubbelink et al. 2014; Campbell et al. 2015; Canu et al. 2015; Gorges et al. 2015; Tan et al. 2015; Guimaraes et al. 2016; Koshimori et al. 2016; Peraza et al. 2017). However, we show disruptions of internetwork somatomotor interactions. These internetwork differences included somatomotor to cortical sensory and association systems and subcortical systems, like the cerebellum and thalamus. Interestingly, lower internetwork FC of the motor system weakly mirrored that found in other sensory systems (visual, auditory, seen also in (Olde Dubbelink et al. 2014; Canu et al. 2015; Guimaraes et al. 2016; Ma et al. 2017; Peraza et al. 2017)). Reduced internetwork FC between somatomotor and sensory networks in PD may relate to reports of impaired sensory processing and sensorimotor integration (Abbruzzese and Berardelli 2003; Patel et al. 2014; Richards et al. 1993), as further suggested by the relationship between visual-cerebellar FC and visuospatial performance reported here.

The large, prominent, intranetwork and internetwork disruptions of the cerebellar and thalamic systems are particularly interesting. Although known to have central roles in motor function as well as important processing and gating roles for many other cortical networks (Alexander et al. 1990; Middleton and Strick 2000; Saalmann and Kastner 2011; Buckner 2013; Hwang et al. 2017), few prior studies comprehensively included these regions in studies of connectome-wide disruption in PD (although for cerebellum see (Onu et al. 2015; Guimaraes et al. 2016; Ma et al. 2017) and for thalamus see (Baggio et al. 2014; Gorges et al. 2015; Vervoort et al. 2016)). Our findings indicate that the cerebellum and thalamus may be key sites of dysfunction in PD. Notably, subregions within the thalamus and cerebellum showed distinct patterns of differences between PD and HC. In the thalamus, disruptions primarily related to frontal association subregions. In contrast, FC was most strongly enhanced in motor subregions of the cerebellum. The significance of these patterns remains unclear, but we speculate that they may reflect disrupted or possibly compensatory interactions between motor and executive control functions. As FC of both the cerebellum and thalamus related to behavioral performance, these structures may play a role in the clinical manifestations of PD.

In contrast to these consistent blockwise differences in FC, association networks exhibited a more variable pattern. Robust reductions in the magnitude of negative correlations occurred between somatomotor and certain control related networks (e.g., frontoparietal and salience), while other association networks showed mixed patterns (e.g., cinguloopercular, ventral attention, and default). Of note, interactions did not differ within or among association networks, likely reflecting the ubiquitous motor deficits and the heterogeneity of cognitive function across our sample of nondemented PD participants, with some cognitively intact and others with cognitive impairment. More focused analyses of specific association networks in a more cognitively impaired PD group may reveal additional network disruptions (Rektorova et al. 2012; Tessitore et al. 2012; Baggio et al. 2014, 2015; Amboni et al. 2015; Gorges et al. 2015; Peraza et al. 2017).

\section{PD Disrupts Networks Downstream of the Primary Sites of Pathology}

The pattern of strongest disruptions-among cortical sensorimotor, thalamic, and cerebellar networks-is intriguing, as it differs from previous research focused on nigral-striatal dopamine pathway degeneration and dysfunction (Helmich et al. 2010; Hacker et al. 2012; Tahmasian et al. 2015). Although PD participants demonstrated reduced striatal FC, consistent with previous reports (Campbell et al. 2015; Hacker et al. 2012), these differences were relatively modest and notably less prominent than FC reductions in other networks.

Cortical $\alpha$-synuclein proteinopathy (i.e., Lewy bodies, neurites, and grains) and abnormal $\beta$-amyloid deposition (Kotzbauer et al. 2012) may contribute locally to network-level FC disruption. However, $\alpha$-synuclein deposition typically progresses from caudal brainstem to the midbrain, and then affects frontal and limbic cortical regions, whereas, sensorimotor cortical regions are among the last to develop Lewy pathology (Braak et al. 2004). Thus, the most prominent network disruptions in our nondemented PD sample, of cortical sensorimotor systems as well as the cerebellum and thalamus, suggest that these disruptions reflect remote, downstream effects since synucleinopathy does not develop in these areas until late stages. This finding is consistent with previous PET blood flow and metabolism studies, which also suggest downstream consequences of neuropathology (Wooten et al. 1982; Perlmutter and Raichle 1985; Eidelberg 1998; Hershey et al. 1998; Sala et al. 2017). In support of this idea, previous studies have demonstrated that proteinopathy may influence sensorimotor network function, suggesting that reduced FC arises as a downstream consequence of pathology in other regions. For example, CSF $\alpha$-synuclein levels relate to sensorimotor network integrity (Campbell et al. 
2015) and CSF $\alpha$-synuclein and $\beta$-amyloid levels relate to a global measure of network disruption (Madhyastha et al. 2015). A related possibility is that FC disruption in PD arises from neurotransmitter deficits, caused by disruptions to dopaminergic, noradrenergic, cholinergic, and serotonergic nuclei (Braak et al. 2004; Kehagia et al. 2010; Halliday et al. 2011; Buddhala et al. 2015b), whose projections impact widespread cortical regions.

We propose that a combination of proteinopathy and neurotransmitter deficits leads to emergent disruptions in network organization. For instance, deficits in brainstem and subcortical nuclei important for motor function may contribute to functional deficits in cortical motor areas through frontostriatal circuitry (Alexander et al. 1990; Middleton and Strick 2000); similarly, deficits in dopaminergic or cholinergic projections may affect the function of control, reward, or sensory systems (Bohnen and Albin 2011; Cools 2006; Muller and Bohnen 2013; Tahmasian et al. 2015). By carefully and comprehensively defining functional network differences in PD, this work lays an important foundation for future studies aimed at understanding the mechanisms underlying FC disruptions in $\mathrm{PD}$, using a combination of multimodal, longitudinal, and interventional approaches.

\section{Network Disruptions Relate to Motor and Cognitive Performance}

Altered network-level FC may relate to the clinical aspects of $\mathrm{PD}$, which include motor, cognitive, and psychiatric features. For example, motor severity correlated with both thalamic FC and the interaction between somatomotor and reward networks. Previous studies also demonstrated links between striatal and motor network FC with motor severity (Hacker et al. 2012). Cognitive deficits also occur with PD, initially affecting attention and executive function, and later include visuospatial and memory deficits as dementia develops (Barker and Williams-Gray 2014; Gasca-Salas et al. 2014). Consistent with these cognitive deficits, we found network deficits related to visuospatial and executive function. Worse performance was primarily associated with lower magnitude (i.e., weaker) negative as well as positive FC. Interestingly, the majority of significant brain-behavior relationships involved internetwork FC, and all involved subcortical network FC, highlighting the importance of these FC differences. These findings indicate that functional network disruptions relate to the heterogeneous pattern of behavioral manifestations in PD. One interesting question is whether FC deficits reflect direct or indirect markers of behavioral disruptions in PD; future work with longitudinal data could delineate the temporal sequence of FC and behavioral deficits to address this question more fully. Moreover, these findings suggest that another exciting avenue for future research will be to examine the links between network alterations and behavioral subtypes in PD.

\section{Strengths and Limitations}

Our robust findings address important methodological issues. The large, well-characterized PD cohort, novel statistical techniques, and comprehensive sampling of cortical and subcortical regions across much of the connectome represent clear strengths. Importantly, our paper is one of few in the field to rigorously account for motion artifacts (see Supplementary Table S1) (Tahmasian et al. 2015); given that PD is a movement disorder, this constitutes a major advance. Furthermore, we replicated findings in split-half samples of the data demonstrating the robustness of these results and the sensitivity of these novel techniques. The large sample size also allowed us to address the potential confounds of sex and education; a subgroup analysis demonstrated that groups matched on sex and education (as well as number and age) resulted in analogous network differences to those found in the full group (Supplementary Fig. S4). Thus, these patient characteristics are unlikely sources of the differences between PD and HC groups. Jointly, these methodological improvements clarify our understanding of the effects of PD on functional networks, addressing the large inconsistencies in results reported in the literature.

Our findings should be qualified by the potential for sleep differences between the PD and HC groups. Resting-state data were collected with eyes-closed, which could encourage sleep during fMRI scans. Sleep systematically modifies FC and may contaminate putative resting state fMRI (Tagliazucchi and Laufs 2014). This may be of particular concern as sleep disturbances (e.g., REM sleep disorder) commonly occur in PD (Comella 2007). We repeatedly emphasized the importance of wakefulness to our participants and we assessed this verbally; scans with overt sleep contamination were removed from analysis. Future studies with concurrent EEG-based sleep monitoring may address this potential confound. Secondly, we restricted our analyses to account for potential atrophy in the PD group, an unaddressed concern in most prior studies of FC in PD (Supplementary Table S1); however, due to this restriction, a few small regions were not included in our analyses (see gaps in Fig. 1). Supplementary Figure S3 includes analyses on a different full set of regions matched for size, demonstrating similar results. Relatedly, while PD pathology impacts brainstem regions, the brainstem is a small structure in which heterogeneous nuclei are packed closely together, and has high susceptibility to pulsation artifacts, presenting a signal quality and spatial resolution challenge for fMRI (Astafiev et al. 2010). Future work targeted at improving measurements from these regions may help to more directly illuminate the relationship between pathology and network dysfunction in PD. Although we demonstrate robust network-level differences using split-half analyses, replication in an independent data set would be ideal. Finally, as the current study is a cross-sectional analysis, we cannot directly measure disease progression; we infer disease progression based on the range of severity of $\mathrm{PD}$ features in this sample and the results of the behavioral correlations. However, future work with longitudinal samples will more directly reveal the course of disease progression within $\mathrm{PD}$.

\section{Conclusions}

We demonstrate that PD is associated with striking "blockwise" disruptions of functional brain networks. Network disruptions-primarily reflecting reduced FC magnitude-were highly selective, affecting blocks of network-to-network interactions, most prominently among cortical sensorimotor, thalamic, and cerebellar networks. These findings suggest that PD may be characterized as a selective "network-level" disease. Furthermore, the most prominent disruptions occurred downstream of the primary neuropathology and pathophysiology in $\mathrm{PD}$, suggesting an emergent nature to the functional network differences. Finally, FC disruptions related to motor and cognitive performance, indicating a potential link to clinical manifestations in PD. 


\section{Supplementary Material}

Supplementary material is available at Cerebral Cortex online.

\section{Funding}

National Institute of Health grants from the National Institute of Neurological Disease and Stroke (NS075321; NS097437; NS41509; 1P30NS098577; and F32NS092290), the National Institute of Aging (AG054405), and the National Institute of Mental Health (K01MH104592), grants from the American Parkinson Disease Association (APDA) Advanced Research Center at Washington University, the St. Louis Chapter of the APDA, Parkinson Study Group and the Parkinson's disease Foundation Mentored Clinical Research Award, American Academy of Neurology and the American Brain Foundation Clinical Research Training Fellowship in Parkinson's disease funded by Abbvie, the Jo Oertli Fund, the Washington University Institute of Clinical and Translational Sciences, and the Barnes Jewish Hospital Foundation (Elliot Stein Family Fund \& Parkinson disease research Fund).

\section{Notes}

Conflict of Interest: W.S. is Founder and President of BioRankings.

\section{References}

Aarsland D, Kramberger MG. 2015. Neuropsychiatric symptoms in Parkinson's disease. J Parkinsons Dis. 5:659-667.

Abbruzzese G, Berardelli A. 2003. Sensorimotor integration in movement disorders. Mov Disord. 18:231-240.

Alexander GE, Crutcher MD, DeLong MR. 1990. Basal gangliathalamocortical circuits: parallel substrates for motor, oculomotor, "prefrontal" and "limbic" functions. Prog Brain Res. 85:119-146.

Amboni M, Tessitore A, Esposito F, Santangelo G, Picillo M, Vitale C, Giordano A, Erro R, de Micco R, Corbo D, et al. 2015. Resting-state functional connectivity associated with mild cognitive impairment in Parkinson's disease. J Neurol. 262: 425-434.

Astafiev SV, Snyder AZ, Shulman GL, Corbetta M. 2010. Comment on "Modafinil shifts human locus coeruleus to low-tonic, high-phasic activity during functional MRI" and "Homeostatic sleep pressure and responses to sustained attention in the suprachiasmatic area". Science. 328:309; author reply 309.

Baggio HC, Sala-Llonch R, Segura B, Marti MJ, Valldeoriola F, Compta Y, Tolosa E, Junque C. 2014. Functional brain networks and cognitive deficits in Parkinson's disease. Hum Brain Mapp. 35:4620-4634.

Baggio HC, Segura B, Sala-Llonch R, Marti MJ, Valldeoriola F, Compta Y, Tolosa E, Junque C. 2015. Cognitive impairment and resting-state network connectivity in Parkinson's disease. Hum Brain Mapp. 36:199-212.

Barker RA, Williams-Gray CH. 2014. Mild cognitive impairment and Parkinson's disease-something to remember. J Parkinson's Dis. 4:651-656.

Biswal B, Yetkin FZ, Haughton VM, Hyde JS. 1995. Functional connectivity in the motor cortex of resting human brain using echo-planar MRI. Magn Reson Med. 34:537-541.

Bohnen NI, Albin RL. 2011. The cholinergic system and Parkinson disease. Behav Brain Res. 221:564-573.

Braak H, Ghebremedhin E, Rub U, Bratzke H, Del Tredici K. 2004. Stages in the development of Parkinson's disease-related pathology. Cell Tissue Res. 318:121-134.
Buckner RL. 2013. The cerebellum and cognitive function: 25 years of insight from anatomy and neuroimaging. Neuron. 80:807-815.

Buckner RL, Snyder AZ, Shannon BJ, LaRossa G, Sachs R, Fotenos AF, Sheline YI, Klunk WE, Mathis CA, Morris JC, et al. 2005. Molecular, structural, and functional characterization of Alzheimer's disease: evidence for a relationship between default activity, amyloid, and memory. J Neurosci. 25:7709-7717.

Buddhala C, Campbell MC, Perlmutter JS, Kotzbauer PT. 2015a. Correlation between decreased CSF alpha-synuclein and Abeta (1)(-)(4)(2) in Parkinson disease. Neurobiol Aging. 36:476-484.

Buddhala C, Loftin SK, Kuley BM, Cairns NJ, Campbell MC, Perlmutter JS, Kotzbauer PT. 2015b. Dopaminergic, serotonergic, and noradrenergic deficits in Parkinson disease. Ann Clin Transl Neurol. 2:949-959.

Burgess GC, Kandala S, Nolan D, Laumann TO, Power JD, Adeyemo B, Harms MP, Petersen SE, Barch DM. 2016. Evaluation of Denoising strategies to address motioncorrelated artifacts in resting-state functional magnetic resonance imaging data from the Human Connectome Project. Brain Connect. 6:669-680.

Campbell MC, Koller JM, Snyder AZ, Buddhala C, Kotzbauer PT, Perlmutter JS. 2015. CSF proteins and resting-state functional connectivity in Parkinson disease. Neurology. 84: 2413-2421.

Canu E, Agosta F, Sarasso E, Volonte MA, Basaia S, Stojkovic T, Stefanova E, Comi G, Falini A, Kostic VS, et al. 2015. Brain structural and functional connectivity in Parkinson's disease with freezing of gait. Hum Brain Mapp. 36:5064-5078.

Chan MY, Park DC, Savalia NK, Petersen SE, Wig GS. 2014. Decreased segregation of brain systems across the healthy adult lifespan. Proc Natl Acad Sci USA. 111:E4997-E5006.

Ciric R, Wolf DH, Power JD, Roalf DR, Baum GL, Ruparel K, Shinohara RT, Elliott MA, Eickhoff SB, Davatzikos C, et al. 2017. Benchmarking of participant-level confound regression strategies for the control of motion artifact in studies of functional connectivity. Neuroimage. 154:174-187.

Comella CL. 2007. Sleep disorders in Parkinson's disease: an overview. Mov Disord. 22(Suppl 17):S367-S373.

Cools R. 2006. Dopaminergic modulation of cognitive functionimplications for L-DOPA treatment in Parkinson's disease. Neurosci Biobehav Rev. 30:1-23.

Diedrichsen J. 2006. A spatially unbiased atlas template of the human cerebellum. Neuroimage. 33:127-138.

Diedrichsen J, Balsters JH, Flavell J, Cussans E, Ramnani N. 2009. A probabilistic MR atlas of the human cerebellum. Neuroimage. 46:39-46.

Eidelberg D. 1998. Functional brain networks in movement disorders. Curr Opin Neurol. 11:319-326.

Fahn S, Elton RL, Committee, M.o.t.U.D. 1987. Unified Parkinson's disease rating scale. In: Fahn S, Marsden CD, Goldstein M, Calne DB, editors. Recent developments in Parkinson's disease. New York: Macmillan. p. 153-163.

Fischl B, Salat DH, Busa E, Albert M, Dieterich M, Haselgrove C, van der Kouwe A, Killiany R, Kennedy D, Klaveness S, et al. 2002. Whole brain segmentation: automated labeling of neuroanatomical structures in the human brain. Neuron. 33: 341-355.

Fischl B, van der Kouwe A, Destrieux C, Halgren E, Segonne F, Salat DH, Busa E, Seidman LJ, Goldstein J, Kennedy D, et al. 2004. Automatically parcellating the human cerebral cortex. Cereb Cortex. 14:11-22. 
Foster ER, Campbell MC, Burack MA, Hartlein J, Flores HP, Cairns NJ, Hershey T, Perlmutter JS. 2010. Amyloid imaging of Lewy body-associated disorders. Mov Disord. 25: 2516-2523.

Fox MD, Snyder AZ, Vincent JL, Corbetta M, Van Essen DC, Raichle ME. 2005. The human brain is intrinsically organized into dynamic, anticorrelated functional networks. Proc Natl Acad Sci USA. 102:9673-9678.

Gasca-Salas C, Estanga A, Clavero P, Aguilar-Palacio I, Gonzalez-Redondo R, Obeso JA, Rodriguez-Oroz MC. 2014. Longitudinal assessment of the pattern of cognitive decline in non-demented patients with advanced Parkinson's disease. J Parkinson's Dis. 4:677-686.

Gordon EM, Laumann TO, Adeyemo B, Huckins JF, Kelley WM, Petersen SE. 2016. Generation and evaluation of a cortical area parcellation from resting-state correlations. Cereb Cortex. 26:288-303.

Gorges M, Muller HP, Lule D, Consortium L, Pinkhardt EH, Ludolph AC, Kassubek J. 2015. To rise and to fall: functional connectivity in cognitively normal and cognitively impaired patients with Parkinson's disease. Neurobiol Aging. 36: 1727-1735.

Gottlich M, Munte TF, Heldmann M, Kasten M, Hagenah J, Kramer UM. 2013. Altered resting state brain networks in Parkinson's disease. PLoS One. 8:e77336.

Greene DJ, Laumann TO, Dubis JW, Ihnen SK, Neta M, Power JD, Pruett JR Jr., Black KJ, Schlaggar BL. 2014. Developmental changes in the organization of functional connections between the basal ganglia and cerebral cortex. J Neurosci. 34:5842-5854.

Guimaraes RP, Arci Santos MC, Dagher A, Campos LS, Azevedo P, Piovesana LG, De Campos BM, Larcher K, Zeighami Y, Scarparo Amato-Filho AC, et al. 2016. Pattern of reduced functional connectivity and structural abnormalities in Parkinson's disease: an exploratory study. Front Neurol. 7:243.

Hacker CD, Perlmutter JS, Criswell SR, Ances BM, Snyder AZ. 2012. Resting state functional connectivity of the striatum in Parkinson's disease. Brain. 135:3699-3711.

Halliday G, Lees A, Stern M. 2011. Milestones in Parkinson's disease-clinical and pathologic features. Mov Disord. 26: 1015-1021.

Helmich RC, Derikx LC, Bakker M, Scheeringa R, Bloem BR, Toni I. 2010. Spatial remapping of cortico-striatal connectivity in Parkinson's disease. CerebCortex. 20:1175-1186.

Hershey T, Black KJ, Stambuk MK, Carl JL, McGee-Minnich LA Perlmutter JS. 1998. Altered thalamic response to levodopa in Parkinson's patients with dopa-induced dyskinesias. Proc Natl Acad Sci USA. 95:12016-12021.

Hwang K, Bertolero MA, Liu WB, D'Esposito M. 2017. The human thalamus is an integrative hub for functional brain networks. J Neurosci. 37:5594-5607.

Jankovic J. 2008. Parkinson's disease: clinical features and diagnosis. J Neurol Neurosurg Psychiatry. 79:368-376.

Johansen-Berg H, Behrens TE, Sillery E, Ciccarelli O, Thompson AJ, Smith SM, Matthews PM. 2005. Functional-anatomical validation and individual variation of diffusion tractography-based segmentation of the human thalamus. Cereb Cortex. 15:31-39.

Kehagia AA, Barker RA, Robbins TW. 2010. Neuropsychological and clinical heterogeneity of cognitive impairment and dementia in patients with Parkinson's disease. Lancet Neurol. 9:1200-1213.

Koshimori Y, Cho SS, Criaud M, Christopher L, Jacobs M, Ghadery C, Coakeley S, Harris M, Mizrahi R, Hamani C, et al.
2016. Disrupted nodal and hub organization account for brain network abnormalities in Parkinson's disease. Front Aging Neurosci. 8:259.

Kotzbauer PT, Cairns NJ, Campbell MC, Willis AW, Racette BA, Tabbal SD, Perlmutter JS. 2012. Pathologic accumulation of alpha-synuclein and Abeta in Parkinson disease patients with dementia. Arch Neurol. 69:1326-1331.

La Rosa PS, Brooks TL, Deych E, Shands B, Prior F, Larson-Prior LJ, Shannon WD. 2016. Gibbs distribution for statistical analysis of graphical data with a sample application to fcMRI brain images. Stat Med. 35:566-580.

La Rosa PS, Shands B, Deych E, Zhou Y, Sodergren E, Weinstock G, Shannon WD. 2012. Statistical object data analysis of taxonomic trees from human microbiome data. PLoS One. 7 e48996.

Lucero C, Campbell MC, Flores H, Maiti B, Perlmutter JS, Foster ER. 2015. Cognitive reserve and beta-amyloid pathology in Parkinson disease. Parkinsonism Relat Disord. 21:899-904.

Ma LY, Chen XD, He Y, Ma HZ, Feng T. 2017. Disrupted brain network hubs in subtype-specific Parkinson's disease. Eur Neurol. 78:200-209.

Madhyastha TM, Askren MK, Zhang J, Leverenz JB, Montine TJ, Grabowski TJ. 2015. Group comparison of spatiotemporal dynamics of intrinsic networks in Parkinson's disease. Brain. 138:2672-2686.

Middleton FA, Strick PL. 2000. Basal ganglia and cerebellar loops: motor and cognitive circuits. Brain Res Brain Res Rev. 31:236-250.

Mintun MA, LaRossa GN, Sheline YI, Dence CS, Lee SY, Mach RH, Klunk WE, Mathis CA, DeKosky ST, Morris JC. 2006. [11C] PIB in a nondemented population: potential antecedent marker of Alzheimer disease. Neurology. 67:446-452.

Muller ML, Bohnen NI. 2013. Cholinergic dysfunction in Parkinson's disease. Curr Neurol Neurosci Rep. 13:377.

Myers PS, McNeely ME, Koller JM, Earhart GM, Campbell MC. 2017. Cerebellar volume and executive function in Parkinson disease with and without freezing of gait. J Parkinson's Dis. 7: 149-157.

Olde Dubbelink KT, Schoonheim MM, Deijen JB, Twisk JW, Barkhof F, Berendse HW. 2014. Functional connectivity and cognitive decline over 3 years in Parkinson disease. Neurology. 83:2046-2053.

Onu M, Badea L, Roceanu A, Tivarus M, Bajenaru O. 2015. Increased connectivity between sensorimotor and attentional areas in Parkinson's disease. Neuroradiology. 57:957-968.

Patel N, Jankovic J, Hallett M. 2014. Sensory aspects of movement disorders. Lancet Neurol. 13:100-112.

Peraza LR, Nesbitt D, Lawson RA, Duncan GW, Yarnall AJ, Khoo TK, Kaiser M, Firbank MJ, O'Brien JT, Barker RA, et al. 2017. Intra- and inter-network functional alterations in Parkinson's disease with mild cognitive impairment. Hum Brain Mapp. 38:1702-1715.

Perlmutter JS, Raichle ME. 1985. Regional blood flow in hemiparkinsonism. Neurology. 35:1127-1134.

Power JD, Barnes KA, Snyder AZ, Schlaggar BL, Petersen SE. 2012. Spurious but systematic correlations in functional connectivity MRI networks arise from subject motion Neuroimage. 59:2142-2154.

Power JD, Cohen AL, Nelson SM, Wig GS, Barnes KA, Church JA, Vogel AC, Laumann TO, Miezin FM, Schlaggar BL, et al. 2011. Functional network organization of the human brain. Neuron. 72:665-678.

Power JD, Mitra A, Laumann TO, Snyder AZ, Schlaggar BL, Petersen SE. 2014. Methods to detect, characterize, and 
remove motion artifact in resting state fMRI. Neuroimage. 84:320-341.

Power JD, Plitt M, Laumann TO, Martin A. 2017. Sources and implications of whole-brain fMRI signals in humans. Neuroimage. 146:609-625.

Power JD, Schlaggar BL, Petersen SE. 2015. Recent progress and outstanding issues in motion correction in resting state fMRI. Neuroimage. 105:536-551.

Rektorova I, Krajcovicova L, Marecek R, Mikl M. 2012. Default mode network connectivity in Parkinson's disease dementia. Eur J Neurol. 19:72-72.

Richards M, Cote LJ, Stern Y. 1993. The relationship between visuospatial ability and perceptual motor function in Parkinson's disease. J Neurol Neurosurg Psychiatry. 56:400-406.

Rosvall M, Bergstrom CT. 2008. Maps of random walks on complex networks reveal community structure. Proc Natl Acad Sci USA. 105:1118-1123.

Saalmann YB, Kastner S. 2011. Cognitive and perceptual functions of the visual thalamus. Neuron. 71:209-223.

Sala A, Caminiti SP, Presotto L, Premi E, Pilotto A, Turrone R, Cosseddu M, Alberici A, Paghera B, Borroni B, et al. 2017. Altered brain metabolic connectivity at multiscale level in early Parkinson's disease. Sci Rep. 7:4256.

Satterthwaite TD, Ciric R, Roalf DR, Davatzikos C, Bassett DS, Wolf DH. 2017. Motion artifact in studies of functional connectivity: characteristics and mitigation strategies. Hum Brain Mapp. doi:10.1002/hbm.23665.

Satterthwaite TD, Wolf DH, Loughead J, Ruparel K, Elliott MA, Hakonarson H, Gur RC, Gur RE. 2012. Impact of in-scanner head motion on multiple measures of functional connectivity: relevance for studies of neurodevelopment in youth. Neuroimage. 60:623-632.

Seeley WW, Crawford RK, Zhou J, Miller BL, Greicius MD. 2009. Neurodegenerative diseases target large-scale human brain networks. Neuron. 62:42-52.

Seitzman BA, Gratton C, Schlaggar BL, Petersen SE, Greene DJ (2017 SFN abstract). An expanded set of regions of interest for functional network analysis: improved representation of the subcortex and cerebellum. In Society for Neuroscience (Washington, DC).

Sperling RA, Aisen PS, Beckett LA, Bennett DA, Craft S, Fagan AM, Iwatsubo T, Jack CR Jr., Kaye J, Montine TJ, et al. 2011. Toward defining the preclinical stages of Alzheimer's disease: recommendations from the National Institute on Aging-Alzheimer's Association workgroups on diagnostic guidelines for Alzheimer's disease. Alzheimers Dement. 7: 280-292.

Tagliazucchi E, Laufs H. 2014. Decoding wakefulness levels from typical fMRI resting-state data reveals reliable drifts between wakefulness and sleep. Neuron. 82: 695-708.

Tahmasian M, Bettray LM, van Eimeren T, Drzezga A, Timmermann L, Eickhoff CR, Eickhoff SB, Eggers C. 2015. A systematic review on the applications of resting-state fMRI in Parkinson's disease: does dopamine replacement therapy play a role? Cortex. 73:80-105.

Tan Y, Tan J, Deng J, Cui W, He H, Yang F, Deng H, Xiao R, Huang Z, Zhang X, et al. 2015. Alteration of basal ganglia and right frontoparietal network in early drug-naive Parkinson's disease during heat pain stimuli and resting state. Front Hum Neurosci. 9:467.

Tessitore A, Esposito F, Vitale C, Santangelo G, Amboni M, Russo A, Corbo D, Cirillo G, Barone P, Tedeschi G. 2012. Default-mode network connectivity in cognitively unimpaired patients with Parkinson disease. Neurology. 79: 2226-2232.

Van Dijk KR, Sabuncu MR, Buckner RL. 2012. The influence of head motion on intrinsic functional connectivity MRI. Neuroimage. 59:431-438.

Vervoort G, Alaerts K, Bengevoord A, Nackaerts E, Heremans E, Vandenberghe W, Nieuwboer A. 2016. Functional connectivity alterations in the motor and fronto-parietal network relate to behavioral heterogeneity in Parkinson's disease. Parkinsonism Relat Disord. 24:48-55.

Wooten GF, DiStefano P, Collins RC. 1982. Regional cerebral glucose utilization during morphine withdrawal in the rat. Proc Natl Acad Sci USA. 79:3360-3364.

Yeo BT, Krienen FM, Sepulcre J, Sabuncu MR, Lashkari D, Hollinshead M, Roffman JL, Smoller JW, Zollei L, Polimeni JR, et al. 2011. The organization of the human cerebral cortex estimated by intrinsic functional connectivity. J Neurophysiol. 106: 1125-1165.

Zhang D, Snyder AZ, Shimony JS, Fox MD, Raichle ME. 2010. Noninvasive functional and structural connectivity mapping of the human thalamocortical system. Cereb Cortex. 20: 1187-1194. 